\title{
Corela
}

Cognition, représentation, langage

HS-1 1 | 2012

RJC Cotexte, contexte, situation

\section{Etude contrastive français / anglais des anaphores lexicales dans la presse et dans la fiction littéraire}

\section{Emmanuel Baumer}

\section{(2) OpenEdition}

\section{Journals}

Édition électronique

URL : http://journals.openedition.org/corela/2233

DOI : $10.4000 /$ corela.2233

ISSN : $1638-573 \mathrm{X}$

Éditeur

Cercle linguistique du Centre et de I'Ouest - CerLICO

Référence électronique

Emmanuel Baumer, «Etude contrastive français / anglais des anaphores lexicales dans la presse et dans la fiction littéraire », Corela [En ligne], HS-11 | 2012, mis en ligne le 02 avril 2012, consulté le 02 mai 2019. URL : http://journals.openedition.org/corela/2233 ; DOI : 10.4000/corela.2233

Ce document a été généré automatiquement le 2 mai 2019.

\section{(c) (i) (2)(2)}

Corela - cognition, représentation, langage est mis à disposition selon les termes de la licence Creative Commons Attribution - Pas d'Utilisation Commerciale - Partage dans les Mêmes Conditions 4.0 International. 


\title{
Etude contrastive français / anglais des anaphores lexicales dans la presse et dans la fiction littéraire
}

\author{
Emmanuel Baumer
}

\section{Introduction}

1 Cette étude porte sur la question éminemment contextuelle des chaînes de référence, c'est-à-dire les séquences d'expressions référentielles renvoyant au même personnage dans un texte donné. Ces chaînes sont principalement constituées de trois types de marqueurs: les noms propres, les pronoms anaphoriques et enfin les syntagmes nominaux anaphoriques que nous appelons ici « anaphores lexicales» (désormais Alex). Graham Ranger (2002), à qui l'appellation est empruntée, donne cette définition: «par anaphore lexicale, nous entendons [un] terme qui, d'une part, fait référence à un antécédent, mais qui, d'autre part, introduit une qualification supplémentaire par le biais d'un élément lexical ».

(1) Eugenio Scalfari, de la presse au roman

Fondateur de «L'Espresso » et de «La Repubblica », le journaliste italien publie une fiction, biographie déguisée de Giovanni Agnelli.

S'il s'est beaucoup promené, Eugenio Scalfari? Allons donc! La question paraît incongrue, appliquée au journaliste le plus célèbre d'Italie, ancien patron de presse, ancien député deTurin et de Milan, aujourd'hui essayiste et romancier, figure de la vie intellectuelle de la Péninsule. Plutôt qu'un amateur de flâneries, ce septuagénaire au regard ferme, à l'élégance raffinée, s'est toujours montré sous les traits d'un homme pressé, enchaînant les livres et les créations de journaux (L'Espresso, en 1955, puis La Reppublica, en 1976), les articles polémiques et les chroniques aiguës. Lui, pourtant, ne sursaute pas le moins du monde. «Mais si, je me suis beaucoup promené, répond-il, sans ironie dans la voix. À l'intérieur de moimême. Et des autres, aussi : quand on dirige un journal de 400 salariés, on se promène forcément dans leur tête, dans leurs espoirs, dans leurs vices. » (Le Monde, emprunté à C. Schnedecker). 
2 C'est plus spécifiquement à ces Alex qu'est consacrée la présente étude. J'ai en effet voulu entreprendre une ébauche de comparaison entre la presse et la fiction littéraire. Il s'agira donc d'analyser la distribution et les fonctions de ces Alex une perspective doublement contrastive : anglais / français et littérature / presse. Pour mener à bien cette analyse, j'ai suivi les chaînes de référence dans deux corpus constitués manuellement. Le premier comporte 40 nouvelles dans chaque langue (pour pouvoir plus facilement étudier les chaînes de référence) et le second 40 articles de presse (dont la plupart sont des portraits mono-référentiels) dans chaque langue. Le cadre théorique adopté est celui de la TOE d'Antoine Culioli, mais nous limiterons ici l'emploi d'une métalangue trop spécifique afin de faciliter la compréhension. Enfin, nous n'aborderons ici, par manque de place, que certains aspects $\mathrm{du}$ problème et les exemples seront surtout tirés du corpus journalistique.

\section{Distribution et constitution des Alex}

Nous commencerons tout d'abord par quelques données statistiques générales pour se donner une idée des phénomènes en jeu, par exemple la distribution :

\begin{tabular}{|l|l|l|l|}
\hline Corpus français & Pronoms & Noms propres & Alex \\
\hline Nouvelles & $65 \%$ & $25 \%$ & $\mathbf{1 0 \%}$ \\
\hline Articles & $60 \%$ & $20 \%$ & $\mathbf{2 0} \%$ \\
\hline
\end{tabular}

\begin{tabular}{|l|l|l|l|}
\hline Corpus anglais & Pronoms & Noms propres & Alex \\
\hline Nouvelles & $70 \%$ & $25 \%$ & $\mathbf{5 \%}$ \\
\hline Articles & $70 \%$ & $27,5 \%$ & $\mathbf{2 , 5} \%$ \\
\hline
\end{tabular}

4 Première constatation: les Alex sont des formes marginales. Dans tous les cas, les pronoms sont largement majoritaires, suivis des noms propres, les Alex représentant toujours la portion congrue. Au demeurant, cela n'est pas très surprenant, car d'autres travaux sur les chaînes de référence ont montré que le pronom pouvait être considéré comme la forme dominante, « non marquée » de la reprise. Catherine Schnedecker (1997) a également bien rendu compte de l'alternance entre nom propre et pronom. Marquant une stabilisation, voire un figement, au niveau quantitatif et qualitatif, le pronom indique que l'on se situe dans une forme de continuité, de stabilité référentielle, alors que le nom propre, lui, implique une sorte de rupture, de « remise à zéro des compteurs référentiels, pour saisir le référent dans un contexte différent ou sans rapport nécessaire avec celui qui précède ».

5 Ceci étant dit, les Alex ne sont pas négligeables pour autant et elles semblent présenter d'importantes différences de distribution en fonction des langues et du genre textuel. $\mathrm{Si}$ l'on regarde les tableaux, on se rend compte que les Alex sont globalement plus nombreuses en français qu'en anglais, mais surtout il y a une différence très importante en ce qui concerne les corpus journalistiques, avec 8 fois plus d'Alex en français qu'en 
anglais (20\% contre seulement 2,5\%). Ceci revient à dire que dans les articles journalistiques de notre corpus anglais, les chaînes de référence sont constituées presque exclusivement de pronoms et de noms propres, comme en (2) :

2) Edward Behr was born on May 71926 in Paris. His parents were of Russian descent, and he had a bilingual education at the Lycée Janson de Sailly and St Paul's School, London. He enlisted in the Indian Army on leaving school, serving in Intelligence in the North-West Frontier from 1944 to 1948 and rising to acting brigade major in the Royal Garhwal Rifles at the age of 22. He then took a degree in History at Magdalene College, Cambridge.

His early career as a reporter was with Reuters in London and Paris. He then had a spell as press officer with Jean Monnet at the European Coal and Steel Community in Luxembourg from 1954 to 1956. Later he joined Time-Life as Paris correspondent, and in the late 1950 s and early 1960 s $\varnothing$ often covered the fighting in the Congo, the civil war in Lebanon as well as the Indo-Chinese border clashes of 1962 . He wrote about the unrest in Ulster, the fighting in Angola and the Moroccan attack on Ifni, the Spanish enclave in West Africa.

Behr was often in Algeria, and in $1961 \varnothing$ published The Algerian Problem. The book had the virtue of being written by a French-speaking outsider with some understanding of, and sympathy for, the positions of both the French and the Algerians. Written when the war was far from over, and going back a century or more over the background, it was considered a fair assessment of a problem which many Frenchmen reckoned no foreigner could possibly understand. The book was said to be compulsory reading at the State Department.

Returning to India for Time magazine, Behr served as bureau chief in New Delhi, travelled in Indo-China, then moved to the mass-circulation American magazine Saturday Evening Post as roving correspondent. In 1965 he went to Newsweek, the weekly news.

Operating from Hong Kong as Asia bureau chief, Behr wrote on China's Cultural Revolution, secured an interview with Mao and reported from Vietnam. The year 1968 turned out to be a hectic one for Behr: he was in Saigon during the Tet offensive, in Paris for the student riots and in Prague when it was occupied by the Russians.

Behr turned gradually from a career in war reporting to writing books and making television documentaries, including award-winning programmes on India, Ireland and the Kennedy family. A notable production was The American Way of Death, Behr's look at America's undertaking industry. (www.telegraph.co.uk, 29/05/2007)

En revanche, en français, les Alex sont en général bien représentées :

3) [Vincent Bolloré] A l'extérieur du groupe, les propos sont moins amènes. Pour se tailler une place dans le PAF, le nouveau venu s'est fait de solides inimitiés. Quelques-unes de plus! Hier, Martin Bouygues (propriétaire de TF 1 et de LCI). Aujourd'hui, Maurice Lévy. Si le président de Publicis salue «l'une des plus belles histoires du capitalisme français ", il se montre cinglant dès qu'il s'agit d'évoquer les méthodes de l'industriel. Voilà un mois, trois pointures de Publicis - Frédéric Raillard, Farid Mokart et, surtout, Christophe Lambert (PDG de Publicis Conseil) ont démissionné sans crier gare pour créer leur propre agence avec l'aide de Bolloré. Lévy n'a pas digéré cet épisode. De quoi hypothéquer, un jour ou l'autre, toute idée d'accord entre Havas et Publicis? «Peut-on raisonnablement s'asseoir à la même table que ce monsieur? Cela pose à mon sens quelques problèmes d'éthique... »Au-delà des querelles, le trouble-fête continue de soulever des questions. En particulier sur la pérennité de ses engagements. Que restera-t-il de ce bel ensemble médiatique dans cinq ou dix ans? Depuis qu'il a rejoint l'entreprise familiale, en 1981, ce patron marginal, dans et hors de l'establishment - conseiller de la Banque de France et redoutable raider - a constamment semblé chercher sa voie, n'hésitant pas à chambouler les activités du groupe. 


\begin{abstract}
Alors, que penser de sa nouvelle marotte? Si les médias représentent désormais une des activités essentielles de la maison (environ $16 \%$ des actifs), ils ne pèsent rien, pour ainsi dire, dans son chiffre d'affaires (moins de $1 \%$ ). Bolloré reste avant tout un conglomérat industriel et financier - un "bric-à-brac ", soutiennent ses détracteurs - présent dans les transports et la distribution d'énergie, mais aussi les films plastiques, les papiers spéciaux ou les batteries électriques. Un gestionnaire encore de multiples intérêts dans l'agro-industrie, notamment des dizaines de milliers d'hectares de plantations exotiques en Asie et en Afrique. Ensuite, Citizen Bolloré fait figure de nain à l'aune de ses concurrents, les Bouygues, Publicis, Murdoch, Lagardère... Enfin, dans les médias, le groupe demeure un acteur $100 \%$ tricolore et le PDG n'entend pas - pour l'heure, du moins - se développer à l'étranger. Même s'il dispose, grâce à la banque d'affaires italienne Mediobanca, dont il est actionnaire, d'une participation dans RCS Media - propriétaire notamment des éditions Flammarion et de fleurons comme le Corriere della Sera et La Gazzetta dello Sport.

Pour la première fois, pourtant, ce touche-à-tout semble mordu. A 54 ans, le chef de clan a, à l'évidence, investi dans les médias dans une optique non seulement patrimoniale, mais aussi successorale. (www.lexpress.fr, 06/02/2007).
\end{abstract}

C'est cette différence fondamentale que nous tenterons d'expliquer par la suite et qui constituera l'essentiel de notre problématique ici, car une comparaison plus exhaustive des autres aspects des Alex dépasserait le cadre imparti.

Que se passe-t-il maintenant au niveau de la structure de ces Alex: On considérera successivement les trois composantes principales de ces groupes nominaux :

- la détermination nominale,

- le substantif tête du GN,

- l'éventuelle complémentation.

Examinons tout d'abord la détermination nominale :

\begin{tabular}{|l|l|l|l|l|}
\hline Corpus français & $\begin{array}{l}\text { Article } \\
\text { défini }\end{array}$ & $\begin{array}{l}\text { Article } \\
\text { indéfini }\end{array}$ & Possessif & $\begin{array}{l}\text { Démons- } \\
\text { tratif }\end{array}$ \\
\hline Nouvelles & $56 \%$ & $7 \%$ & $33 \%$ & $4 \%$ \\
\hline Articles & $83 \%$ & $8 \%$ & $3 \%$ & $6 \%$ \\
\hline
\end{tabular}

\begin{tabular}{|l|l|l|l|l|}
\hline Corpus anglais & $\begin{array}{l}\text { Article } \\
\text { défini }\end{array}$ & $\begin{array}{l}\text { Article } \\
\text { indéfini }\end{array}$ & Possessif & $\begin{array}{l}\text { Démons- } \\
\text { tratif }\end{array}$ \\
\hline Nouvelles & $60 \%$ & $10 \%$ & $25 \%$ & $5 \%$ \\
\hline Articles & $73 \%$ & $4 \%$ & $3 \%$ & $20 \%$ \\
\hline
\end{tabular}

8 Globalement, les marqueurs de forte détermination (génitif, fléchage) sont dominants, ce qui est normal, puisque les Alex sont des formes de reprise avec identification d'un même référent. L'article défini est toujours largement majoritaire. Concernant le corpus littéraire, la distribution des déterminants est étonnamment proche en anglais et en français, ce qui suggérerait une forte influence du genre textuel. Par rapport au corpus de presse, les possessifs sont assez nombreux: par une opération de localisation, ils permettent de repérer les personnages les uns par rapport aux autres (ex : son frère) et 
souvent de donner des indices sur la focalisation (point de vue privilégié). Les articles indéfinis sont beaucoup moins présents : ils correspondent souvent au premier élément d'une chaîne (première mention d'un personnage dans l'espace textuel) ou bien à la réintroducion du même personnage sous un point de vue différent (créant par là même une nouvelle chaîne). Enfin, les démonstratifs, qui sont souvent la trace d'une appréciation de l'énonciateur-narrateur ou d'un personnage (par exemple au style indirect libre) sont statistiquement assez rares. En revanche, dans le corpus journalistique, on peut remarquer que les démonstratifs sont beaucoup plus présents en français.

Regardons à présent les têtes lexicales en jeu :

\begin{tabular}{|l|l|l|}
\hline Corpus anglais & $\begin{array}{l}\text { Propriété } \\
\text { permanente }\end{array}$ & $\begin{array}{l}\text { Propriété } \\
\text { provisoire }\end{array}$ \\
\hline Nouvelles & $84 \%$ & $16 \%$ \\
\hline Articles & $95 \%$ & $5 \%$ \\
\hline
\end{tabular}

\begin{tabular}{|l|l|l|}
\hline Corpus français & $\begin{array}{l}\text { Propriété } \\
\text { permanente }\end{array}$ & $\begin{array}{l}\text { Propriété } \\
\text { provisoire }\end{array}$ \\
\hline Nouvelles & $85 \%$ & $15 \%$ \\
\hline Articles & $75 \%$ & $25 \%$ \\
\hline
\end{tabular}

9 On peut classer ces différents substantifs en fonction du type de propriété qu'ils apportent au référent. Deux principales catégories ont été distinguées. La première correspond à une attribution de propriété stable, définitoire et valable de façon "permanente », hors situation, pour caractériser le personnage : hyperonyme du type homme / femme, terme évoquant la profession ou la fonction, les liens familiaux, etc.... La seconde, en revanche, est liée à un contexte ou un point de vue particulier et ne caractérise le personnage que de façon « provisoire » (personnages jouant plusieurs rôles, point de vue limité d'un personnage, propriété valable seulement dans un cadre temporel restreint.

Dans le corpus littéraire, que ce soit en anglais ou en français, les substantifs évoquant des propriétés permanentes sont largement dominants et se répartissent en trois souscatégories correspondant environ à $1 / 3$ chacune : hyperonyme, fonction, famille. Dans la presse aussi, les propriétés permanentes sont majoritaires, mais le type d'information est en général beaucoup plus diversifié et on peut remarquer que les propriétés contextuelles ou provisoires sont plutôt la marque des articles français.

11 Enfin, en ce qui concerne les compléments des Alex, on retrouve plus souvent une forme de complémentation (adjectifs, groupes prépositionnels, subordonnées relatives, etc...) dans la presse que dans la fiction et les adjectifs employés y sont plus fréquemment subjectifs, porteurs d'une appréciation ou d'une valuation. Venons-en maintenant à la fonction de ces Alex. 


\section{Fonction(s) des Alex}

12 Par nature, les Alex sont des formes à double vocation : il s'agit d'anaphores, donc leur fonction première = renvoi au même élément, maintien de la référence, mais aussi apport de qualification par le biais de l'élément lexical et les éventuels compléments à valeur qualitative. Culioli (1990) les note avec la pondération suivante : qlt (qnt).

(Qnt) = au second plan, maintien de l'occurrence spatio-temporelle,

Qlt = prépondérant, qualification additionnelle, ajout de propriétés.

13 Cependant quel est leur fonctionnement précis dans les enchaînements textuels et selon les genres différents?

Corpus de nouvelles : 4 fonctions principales

Globalement, on retrouve les mêmes fonctions dans le corpus littéraire en anglais et en français : fonction principale désignation du personnage.

-1 / construction référentielle (en 1ère mention) puis désignation dans les reprises.

- 2 / structuration textuelle et discursive. Comme le Npr, les Alex semblent jouer un rôle dans la structuration du texte. Placement dans des espaces - charnières : après une rupture temporelle, spatiale, argumentative ou autre, ou bien en début de paragraphe encore plus marqué en français $=40 \%$ en anglais, $65 \%$ en français.

- 3 / dissipation de l'ambigüité référentielle ( $30 \%$ des cas) : dans un contexte ou une ambigüité référentielle serait possible (impossibilité de substituer un pronom sans risque de confusion).

- 4 / expression (implicite) du point de vue: (perspective du narrateur ou des personnages, par exemple description d'un personnage selon la perception d'un autre, commentaire du narrateur (le malheureux garçon, etc.)

Corpus de presse: Ici, la fonction principale serait plutôt l'apport informatif, la qualification du personnage, plutôt que la désignation, d'où la présence plus fréquente de compléments à valeur qualitative déjà remarquée. (Particulièrement dans les portraits : contenu nouveau, différentes informations sur le personnage).

Revenons à présent à la dissymétrie déjà observée, c'est-à-dire le fait qu'il semble y avoir beaucoup moins d'anaphores lexicales en anglais qu'en français dans le discours journalistique.

17 Comment peut-on interpréter ce net contraste? Ranger (2002) propose une hypothèse fondée sur le genre grammatical. Le français ne disposant que de deux formes (il / elle) alors que l'anglais en a trois (he / she et le pronom neutre it), les nombreuses anaphores lexicales serviraient par exemple à désambiguïser des interférences potentielles entre des référents animés et inanimés. S'il est vrai que la concurrence entre plusieurs objets textuels - et plutôt entre différents référents animés humains, car en observant les données contextuelles la confusion animé/inanimé est rarement possible - peut parfois justifier l'emploi de formes de reprise avec un rôle ponctuel de désambiguïsation, cette analyse ne suffit pas à expliquer le phénomène, puisque dans les textes quasi-monoréférentiels de notre corpus (où les cas d'ambigüité potentielle ne dépassent pas $10 \%$, contrairement au corpus littéraire), les anaphores lexicales abondent en français.

Mais on se rend surtout compte que l'anglais se limite la plupart du temps à un certain type de reprises lexicales, qui correspondent en fait à une caractérisation relativement «objective». Les informations apportées concernent principalement la fonction (the president, the singer, the artist), l'origine géographique (the Frenchman, the East 
Londoner), les relations familiales (his wife, this mother of four), l'âge (the 24 year old), ou le physique (a tall, silver-haired figure). La liste n'est pas exhaustive, mais en anglais on reste presque toujours dans ce type d'apport informatif relativement objectif.

On retrouve bien évidemment ces descriptions « neutres » en français, mais les anaphores lexicales semblent avoir une fonction beaucoup plus large, avec par exemples des compléments nettement plus subjectifs ( le chercheur déjanté, cette élégante au verbe clair, ce cardinal tout-terrain, le poète incandescent", etc.). Ceci révèle ainsi des stratégies de présentation de l'information assez différentes dans les deux langues. En anglais, même dans des articles très critiques (ex: portraits satiriques de George Bush, Tony Blair, etc.) la subjectivité est véhiculée par d'autres moyens. A nos yeux, ce qui distingue véritablement l'utilisation des anaphores lexicales du français peut être résumé en trois points :

\section{1 une présentation condensée de l'information}

20 Le français a beaucoup plus souvent recours à des Alex, souvent introduites par des démonstratifs. Elles viennent apporter des éléments d'information nouveaux sur les personnages décrits, tout en maintenant le lien référentiel, ce qui permet de condenser la structuration de l'information:

4) [Jean-Pierre Cassel] C'est à Saint-Germain-des-Prés, dans l'une de ces caves où l'on swinguait à la fin des années 1950, que ce grand type élégant s'est révélé aux autres et à lui-même. Un soir, ce fou de jazz et de claquettes est repéré par Gene Kelly. (Le Monde, 21/04/2007)

En effet, on garde ainsi la référence tout en limitant le nombre de prédications (du type : JP Cassel est un grand type élégant / il est fou de jazz et de claquettes. En anglais, cette forme d'économie linguistique passe plutôt par le recours aux appositions. De plus, nous avons vu que ces syntagmes démonstratifs ou définis sont très souvent accompagnés d'une expansion à valeur qualitative, par exemple une subordonnée relative qui permet de fusionner deux prédications

5) [Jean-Claude Brialy] ce quêteur du temps perdu qui avait encore eu la chance de fréquenter les plus grands artistes du siècle dernier. (www.telerama.fr)

\section{2 une forte dépendance contextuelle}

22 Alors qu'en anglais la plupart de ces formes de reprises sont indépendantes du contexte et pourraient renvoyer au référent en toute circonstance, la distribution de ces formes de reprise en français révèle qu'une bonne partie de celles-ci nécessitent une préconstruction en amont (dans le contexte gauche) pour pouvoir être interprétées. Les propriétés attribuées peuvent donc dépendre uniquement du contexte et ne pas qualifier le sujet de façon stable, définitoire. Il faut ainsi un frayage discursif pour reconstituer le lien référentiel. Voici quelques exemples de ce phénomène très répandu en français et beaucoup moins productif en anglais :

6) Rafael Nadal est au bord du gouffre face à Jarkko Nieminen en quarts de finale (4-6, 1-4) avant de se reprendre. "Oui, bien sûr que j'ai eu peur, reconnaîtra le miraculé » (www.lequipe.fr).

7) Nadal était bel et bien déstabilisé par la montée en régime époustouflante du Suisse. Car le diesel helvète avait définitivement pris la mesure de l'événement. Le tombeur de Carlos Moya en demi-finales (7-6, 4-6, 7-5) en avait effectivement gardé sous le pied et ne tremblait pas sur sa mise en jeu (2-6, 4-1). L'écart était fait, la 
deuxième manche pouvait logiquement tomber dans son escarcelle. Mais un champion comme Federer ne se contente pas, quand il le peut, de gagner. Non, lui souhaitait triompher ce dimanche et concluait le set sur le service de l'Espagnol, après plusieurs avantages infructueux (2-6, 6-2). (www.lequipe.fr)

D'autre part, quand l'anglais utilise ce genre de reprises, la cohésion textuelle est presque systématiquement maintenue par des relations sous-jacentes de cause, d'opposition, etc.

8) In 1997 a haitian man named Abner Louima was sodomized with a mop handle by a cop in a Brooklyn-precinct bathroom. Two years later, an unarmed street peddler named Amadou Diallo was killed when police in the Bronx fired 41 shots at him in a dark vestibule. And a year after that, an unarmed security guard named Patrick Dorismond, who had been trying to hail a cab outside a midtown bar, was shot to death after a scuffle with undercover cops. Giuliani denounced the cops who brutalized Louima but defiantly backed those who killed Diallo and Dorismond. (In those cases, juries cleared the officers of wrongdoing.) After Dorismond was killed, Giuliani's instinct to defend the police led him to attack the unarmed victim; the mayor authorized release of Dorismond's juvenile records to "prove" his propensity for violence. The dead, Giuliani argued, waive their right to privacy. Even old friends and supporters were appalled. The man who had saved New York City (ctx) saw his job-approval rating drop to $32 \%$. ( = relation d'opposition)

9) First, this daughter of an army colonel argued for a clamp-down on teenage criminals. ( = relation de cause)

...alors qu'en français, malgré la proportion de cas fortement liés au contexte, on retrouve parfois des anaphores lexicales dont la pertinence est parfois discutable (en dehors de l'apport de qualification) :

10) [Daniel Libeskind] Fils d'immigrés juifs polonais, cet architecte a remporté le concours pour la reconstruction du World Trade Center. Né en Pologne après la Seconde Guerre Mondiale, Daniel Libeskind y a vécu l'antisémitisme du régime communiste après que ses parents avaient subi celui des nazis. Sa famille a rapidement émigré en Israël, où elle s'est vite lassée de la vie dans les kibboutzim avant de partir pour les États Unis. De quoi donner le goût du nomadisme - avec quatorze déménagements en trente-cinq ans! - à ce virtuose de l'accordéon! (emprunté à Schnedecker 2006)

\section{3 enfin et surtout : une qualification subjective « masquée »}

Un certain nombre d'anaphores lexicales correspondent à ce que l'on pourrait appeler une qualification du personnage filtrée par la subjectivité du journaliste ou véhiculée par la mémoire interdiscursive médiatique (cf., entre autres, Sophie Moirand (2007)), qui introduit parfois un jugement modal appréciatif. Néanmoins, le fait d'intégrer cette qualification dans une forme de nominalisation, par définition stabilisée et même préconstruite, ainsi que l'absence de prédication verbale (l'identification avec le verbe ETRE restant simplement sous-jacente) permettent dans une certaine mesure de masquer, voire de gommer la prise en charge énonciative de ces anaphores et donc de rendre ces jugements appréciatifs moins réfutables par le co-énonciateur. C'est donc finalement en discours que l'énonciateur construit cette qualification subjective du référent, tout en s'effaçant derrière les anaphores lexicales. Le lecteur des articles reçoit de fait ces informations subjectives sans pouvoir clairement identifier la source du jugement et peut donc moins facilement les réfuter. Dans les énoncés suivants, on peut remarquer les traces de jugement appréciatif dans les différentes anaphores lexicales : par exemple sale gosse en (11), fausse héritière en (9), fin observateur en (14) l'appellation mégalo-parano en (13) : 
11) [Le prince Harry] : Soixante ans après la libération des camps, le fils cadet de Charles et Diana n'a rien trouvé de mieux que de se déguiser en soldat nazi. Le énième faux pas du sale gosse de Buckingham, et un nouveau scandale pour une monarchie décriée. (lexpress.fr)

12) [Ségolène Royal] Cette fausse héritière de Mai, véritable fille du pompidolisme ou du giscardisme formaté par Science Po et l'Ecole Nationale d'Administration, n'a pas d'idées, pas de programme. [...] Dans Libération de samedi, Henri Emmanuelli appelle de ses vœux la construction d'un pôle de gauche - «un grand parti progressiste avec les antilibéraux »-: je n'exclus donc pas de re-voter un jour pour le Parti Socialiste, mais quand il sera redevenu de gauche. Pas envie de voter pour Giscard, fût-il en jupon...(blog M. Onfray)

13) [Michel Polnareff] Espéré, annoncé et démenti, ce retour en terre natale du mégalo-parano fait l'événement de ce mois de mars, après avoir nourri maintes rumeurs et fait couler beaucoup d'encre. (evene.fr)

14) [Jean-Claude Brialy] Il y avait quelque chose de proustien chez ce fin observateur des arts et des lettres [...]. (telerama.fr)

En anglais, en revanche, ce type de qualification, quand elle apparait, passe le plus souvent par d'autres moyens linguistiques, soit, de façon assez minoritaire, par des prédications en BE prises en charge par l'énonciateur, soit, cas de loin le plus fréquent, elles sont attribuées à une instance différenciée de l'énonciateur (avec emploi du passif sans complément d'agent : he's said to be, he's been branded / hailed / billed / described / dubbed / admired as, ce qui permet d'occulter l'origine de la qualification, ou bien avec mention plus ou moins explicite de la source du jugement: his opponents say, he's considered by many, etc.). Les exemples comme (15) sont de loin les plus fréquents en anglais :

15) [Vonnegut] Described by Gore Vidal as the worst American writer ever, he was none the less at one point said to be the most widely taught in American universities.

\section{BIBLIOGRAPHIE}

CHASTAIN, C. (1975) « Reference and context », in Language, Mind and Knowledge, Gunderson, K., (ed.), University of Minnesota Press, Minneapolis, pp. 194-269.

CHAROLLES, M. (1994) «Cohésion, cohérence et pertinence du discours », in Travaux de linguistique vol. 29, pp. 125-151.

CORBLIN, F. (1995) Les formes de reprise dans le discours, Presses Universitaires de Rennes.

CULIOLI, A. (1990) Pour une linguistique de l'énonciation 1 : opérations et représentations, Gap : Ophrys.

CORNISH, F. (1998) « Les "chaînes topicales" : leur rôle dans la gestion et la structuration du discours », in Cahiers de Grammaire 23, pp. 19-40.

JENKINS, C. (2002) « Les procédés référentiels dans les portraits journalistiques », XV Skandinaviske romanistkongress, Oslo, 12-17 août 2002.

KLEIBER, G. (1994) Anaphores et pronoms, Louvain-la-Neuve, Duculot. 
MOIRAND, S. (2007) « Discours, mémoires et contextes : à propos du fonctionnement de l'allusion dans la presse », in : Corela, Numéros spéciaux, Cognition, discours, contextes.

RABATEL, A. (2004) «Quand voir c'est (faire) penser. Motivation des chaînes anaphoriques et point de vue », in Figures de la lecture et du lecteur, Cahiers de Narratologie 11, www.revel.unice.fr.

RANGER, G. (2002) « Notes sur la traduction de l'anaphore lexicale du français vers l'anglais », in Langues et cultures en contact. Traduire e(s)t commenter, Catherine Paulin (ed.), Presses universitaires de Franche-Comté, pp. 83-99.

SALLES, M. (2006) « Cohésion-cohérence : accords et désaccords », in Corela, Numéros spéciaux, Organisation des textes et cohérence des discours.

SCHNEDECKER, C. (1997) Nom propre et chaînes de référence. Paris, Klincksieck.

SCHNEDECKER, C. (2005) « Les chaînes de référence dans les portraits journalistiques : éléments de description », in Travaux de linguistique 51, pp. 85-133.

SCHNEDECKER, C. (2006) « Anaphores prédicatives démonstratives : de la cohésion syntagmatique à la cohérence textuelle ». Corela, Numéros spéciaux, Organisation des textes et cohérence des discours.

\section{RÉSUMÉS}

Dans ce article, j'ai tenté de dégager les similitudes et les contrastes dans les chaînes anaphoriques reprenant un nom propre, en fonction notamment du genre textuel (fiction littéraire / presse) et de nombreux paramètres contextuels. Lorsque l'on compare ces chaînes anaphoriques en anglais et en français, on peut observer des différences relativement systématiques entre les deux langues, principalement en ce qui concerne les anaphores lexicales.

In this article, I tried to find out the similarities and the differences in the referential chains referring to a proper noun, according to textual genres (literary fiction / newspaper articles) and many other contextual parameters. When those referential chains in french and in english are compared, reccurring differences can be noticed, mainly as far as lexical anaphora are concerned.

\section{INDEX}

Keywords : anaphora, proper noun, referential chains, pre-construction

Mots-clés : noms propre, anaphore, chaînes de référence, qualification, préconstruction

\section{AUTEUR}

\section{EMMANUEL BAUMER}

Université Sophia Antipolis, Nice / Université Paris-Diderot 